\title{
The Flipped Classroom and Simulation: a Primer for Simulation Educators
}

\author{
Chaoyan Dong ${ }^{1}$ (1) $\cdot$ John L. Szarek ${ }^{2} \cdot$ Trent Reed $^{3}$ \\ Published online: 3 August 2020 \\ (C) International Association of Medical Science Educators 2020
}

\begin{abstract}
The flipped classroom has become part of the lexicon in discussion of pedagogies in healthcare education. When reviewing the simulation literature, however, the term is only recently being mentioned. We determined there was a need for this article based on workshops held at the annual International Meeting for Simulation in Healthcare (IMSH), which were facilitated by the authors. In the workshops, most of the participants had heard the term and had first-hand experience with the flipped model in simulation; however, there were varied interpretations of the flipped model and several concerns related to implementation of the model. In this article, we define the flipped classroom model, review the existing work on using the flipped model in healthcare education, and provide five recommendations regarding use of the flipped model in simulation. The framework for summarizing the work and presenting the recommendations is based on a brief review of the literature and from the participant discussions at the IMSH workshops.
\end{abstract}

Keywords Flipped classroom $\cdot$ Simulation $\cdot$ Healthcare education

\section{Background}

The flipped classroom is a pedagogical model in which preclass activities are emphasized and typical in-class lectures are replaced with student-centered activities [1]. The flipped classroom has become part of the lexicon in discussion of pedagogies in healthcare education. When reviewing the simulation literature, however, the term is rarely found. For instance, the term does not appear in the Healthcare Simulation Dictionary [2] published by the Society for Simulation in Healthcare and a search for keywords "flipped classroom" in the Society's journal revealed only one article directed toward the flipped classroom and simulation, which was by one of the co-authors of this commentary (TR). A similar search in

Chaoyan Dong

dong.chaoyan@skh.com.sg

1 Sengkang General Hospital, 110 Sengkang East Way, Singapore 544886, Singapore

2 Clinical Pharmacology, Geisinger Commonwealth School of Medicine, Scranton, PA, USA

3 Department of Emergency Medicine and Medical Education, Simulation Education, Stritch School of Medicine, Loyola University Medical Center, Loyola University Chicago, Chicago, IL, USA
PubMed yielded only a few studies which will be discussed below. At the International Meeting on Simulation in Healthcare (IMSH), we (the authors of the article) conducted workshops titled Using the Flipped Classroom Model in Simulation-Based Medical Education. The majority of the participants had heard the term and had first-hand experience with the flipped model in simulation; however, there were varied interpretations of the flipped model and several concerns related to implementation of the model.

The flipped classroom's emphasis on students completing the course materials before the face-to-face session coincides with simulation education standards defined by professional bodies. For example, the International Nursing Association for Clinical Simulation and Learning (INACSL) emphasizes the importance of preparation activities before simulation in criterion 10 of the standard related to simulation design [3]: specifically, "Design and develop preparation activities and resources to promote the best possible opportunity for participants to be successful in addressing the simulation objectives" [3] and "Allow for participants to complete preparation activities in advance of the simulation prebriefing" [3]. However, in reality, busy health professions students often attend simulation sessions without reviewing or doing preparation activities assigned by faculty, which hinders achievement of the intended learning outcomes [3]. Even though 
simulation professional bodies advocate for the pre-simulation activities, not much work has been done in simulation community regarding how to best motivate students to complete preparation work, which we suggest can be achieved by integrating the flipped model.

In this commentary, we review existing studies using the flipped model in healthcare education, including the few integrating the flipped model in simulation, discuss how the flipped model may help to overcome barriers and challenges in medical simulation, and provide recommendations regarding use of the flipped model in simulation. The framework for summarizing the work and presenting the recommendations is based on a literature review on the flipped model in medical education and participant discussions from our IMSH workshops conducted at the annual meetings in 2016 and 2017.

\section{Brief Review of the Flipped Classroom}

The flipped classroom emphasizes the importance of preparation activities, which might consist of readings, videos, podcasts, or games. By doing so, students learn the material beforehand. In the subsequent face-to-face setting, knowledge gained before the class is applied in various activities, such as case-based discussion, peer instruction, think-pair-share, audience response questions, collaborative learning, and simulations. A meta-analysis by Freeman et al. [4] found that course failure rates were $55 \%$ higher for traditional lecture courses as opposed to courses involving active learning. More specifically to the flipped model, a number of studies demonstrate better examination performance by students in flipped classrooms compared with those in traditional lecture-based classes across a variety of health professions [5-7]. The flipped classroom provides a more studentcentered method, enhances student engagement, and improves intended learning outcomes [8,9]. A recent systematic review concludes the flipped model is a promising teaching approach to increase learners' motivation and engagement, but calls for more solid evidence on its effect on changes in knowledge and skills as well as its long-term effects on professional practice and patient care [10]. Overall published evidence on the impact of the flipped classroom model justifies its use $[11,12]$.

In simulation, there have been only a few studies investigating the impact of using the flipped model. For example, Reed and colleagues [13] used the flipped simulation training model with senior medical students during their emergency medicine (EM) clerkship and found that simulation-based mastery learning using asynchronous pre-simulation work and related quizzes for knowledge acquisition prior to simulation training is an effective way for senior medical students to learn and retain EM clinical skills. Reed and colleagues [14] also used a flipped simulation training model for interprofessional education with nursing and medical students which demonstrated a significant impact on self-efficacy and also TeamSTEPPS knowledge and team performance in a simulated patient care environment. Although this research did not isolate the impact of flipped simulation versus other methods, it does provide a framework for successfully integrating the flipped classroom approach in simulation.

Several recent studies and commentaries further support the notion that using a flipped classroom approach in simulation is effective in skill and knowledge acquisition. Liu et al. [15] and Liebert et al. [16] examined the effect of viewing instructional videos had on acquisition of surgical skills before the hands-on sessions. Using a pre-test/post-test design, Liu et al. [15] found that procedural skills and confidence of first- and second-year dermatology residents improved after viewing instructional videos and participating in simulation. Similarly, Liebert et al. [16] showed that medical students in a surgery clerkship had higher post-test scores in each of the 6 modules and that their interest in surgery as a career increased. When compared with a historical cohort, however, there were no differences in NBME Surgery Shelf Examination scores suggesting that the flipped classroom model for simulation is at least an equally effective curricular method. Uther et al. [17] also used a pre-test/post-test design and showed that medical students early in their education demonstrated short-term gains in pediatric knowledge. In this study, they also showed that students in the flipped model demonstrated long-term retention of knowledge compared with a retrospective cohort [17]. Finally, Chiu et al. [18] showed in a head-to-head comparison that the performance of medical students in laparoscopic suturing and knot-tying was enhanced in the flipped classroom approach in simulation compared with traditional methods.

Thus, there is growing evidence that the flipped classroom model is effective in motivating students to complete the preparation activities and enhancing student learning, so educators should not be reluctant to embrace the flipped model in simulation. Riley [19] has gone so far as to say that the flipped classroom model with simulation should be mandated for osteopathic medical students. But what are the best practices related to applying this model to simulation? We provide five recommendations and associated best practices derived from participant discussions during our workshops held at IMSH 2016 and IMSH 2017, and from a brief review of the literature (in particular [20, 21]).

\section{Recommendation 1: the Learning Objectives Should Dictate the Use of the Flipped Model in Simulation}

Whether to use the flipped model for a simulation session depends on the learning objectives of the session. For example, if the goal is for students to learn technical skills such as airway 
management, the knowledge base for the procedural skills training can be delivered through the flipped model. Students can learn or refresh their knowledge of the procedure before the simulation session. The act of retrieving prior knowledge for a simulation session facilitates learning because recalling prior knowledge strengthens students' understanding about the topic [22]. As a second example, if the goal is for students to practice history taking or physical examination with standardized patients, it is important for students to get as much hands-on experience as possible; even so, gaining a knowledge base on the topic is important. This information can be delivered ahead of time, asynchronously, in a flipped model so students can review the material as long or as little as needed to secure an understanding prior to application. Even learning interprofessional and cognitive non-technical skills can benefit from the flipped model by establishing a baseline understanding prior to engaging in simulation activities related to the topic. However, if the topic is new and the learning materials are too difficult for students, the flipped model will not work well as students may feel overwhelmed by reviewing the new content without a clinical base to anchor the information [23]. They may benefit more from a lecture followed with simulation for demonstration and practice together in the simulation lab.

If simulation session objectives lend themselves to the application of the flipped model, it is important to ensure all the activities in the flipped classroom session, including the preparation work and the simulation, align with the overall learning objectives. This alignment will highlight key learning points, ensure that relevant baseline knowledge is established through the pre-simulation work, and allow the focus of the face-to-face time to be dedicated toward higher levels of cognition along Bloom's Taxonomy [24] such as knowledge application, skills practice, and demonstration.

\section{Recommendation 2: Preparation Work Should Be Engaging and Effective so Learners Can Acquire Baseline Knowledge Through Various Techniques Emphasized in the Flipped Model}

The flipped model emphasizes the importance of the preparation work which refers to assigned materials that students are expected to complete prior to the simulation activity [21]. During the simulation session, students are required to retrieve knowledge or skills acquired through preparation work and apply it in the form of active learning [9]. This requires that students take ownership in their learning by completing the preparation work before coming to the simulation session which helps them identify learning gaps. If a student comes in unprepared, they could have a negative impact on their peers in the same session and may require faculty to review the content separately, reducing the time for simulation and debriefing.
To ensure that participants complete the preparation work, the information should be presented in an engaging and interactive format to keep students interested in reviewing the materials. For example, alternatives to assigning articles to read as preparation work include presenting in audio, video, or game format. Preparation work can also be in the format of case reviews in which the cases come from other parts of the curricula to help students see curricular connections. One unique example of preparation work makes use of recorded simulation sessions. One of the co-authors of this commentary (CD) and colleagues experimented with recording year 3 nursing and midwifery students' performance in simulated acute care cases and using those videos as preparation work for year 2 students in the same disciplines to watch before their simulation sessions. The preliminary results show that watching the videos improved year 2 students' knowledge and skills in resuscitation.

Adequate student engagement and participation in the preparation activities allow faculty to be more efficient in managing the face-to-face time. With a baseline knowledge established through completing the preparation work, faculty can spend more time debriefing, rather than elaborating on background knowledge, since debriefing improves learning outcomes [25-27]. With proper preparation, students are more likely to show up with confidence and be more engaged in the simulation activities. The preparation work serves as a great resource for those students requiring extra time to understand a topic since they have the option of spending more time with the material based on their individual needs.

Creating an opportunity for high-quality learner-centered preparation work with the flipped model is helpful in general but appears to be particularly promising for interprofessional activities. It helps learners feel they are entering the simulation on even ground with other professionals as it relates to the planned simulation session [27].

Utilizing advanced technology formats to host the presimulation materials can be daunting for some faculty who lack that expertise. With this potential challenge in mind, we suggest faculty members work with technology staff and/or instructional design professionals in their institution. This will help motivate faculty to use the approach [20]. In addition, there are credible teaching resources available on the Internet so faculty can curate online information which makes the process less burdensome.

\section{Recommendation 3: Students Should Be Held Accountable for Completion of Preparation Work}

Preparation work is part of the flipped classroom approach but particularly during simulation, completion of preparation work is often not enforced. For example, in several of the 
studies summarized above, students were expected to do the preparation work but there was little to no monitoring of whether the students actually did the work $[15,18,19]$. In one of the studies in which the student viewing of online video content was monitored, only $69 \%$ of students watched all of the videos [15]. Our assertion is that completing the preparatory work is critical to the learning process in this model; therefore, learners should be held accountable for completion. Multiple methods exist to ensure students complete the preparation work when employing the flipped classroom approach. The most common option utilized is a quiz, which can either be integrated into the preparation work or follow it. Quizzes allow students to self-assess their grasp on the material and stimulate further review to fill knowledge gaps. Students' scores from the quizzes can be counted as part of their simulation performance if the session is intended for a summative purpose or can be part of the participation grade if the simulation is solely formative. Assessment drives learning [28], and this will direct their attention during simulation and debriefing.

As an example, a quiz may follow an e-module and successful completion of the quiz may result in a certificate of completion. A learner may take a screenshot of this certificate or print it and bring it to the simulation session as an admission ticket for participation. If they do not do the preparation work, then they do not get credit for participation. As another example of the use of quizzes, a school may choose to host quizzes in a learning management system. If the curriculum is competency based then, following a videocast, students may be required to take a quiz and obtain a passing score within the learning management system in order to participate in the face-to-face simulation session.

Other activities exist to hold learners accountable for preparation work including student presentations and case discussions. Students can deliver brief presentations as evidence of completion and grasp of the relevant topics. Case discussions can precede simulation as a way to assure necessary knowledge base is obtained. As a final example, peer evaluations can be used as a source of preparation incentive. One of the co-authors of this primer (JS) uses peer evaluations in their systems-based basic science courses for second year medical students as a means of holding students accountable for the pre-work in the courses [29].

\section{Recommendation 4: Flipped Simulation Should Not Stand Alone but Instead Integrate into the Larger Curricula}

Faculty should have a clear understanding of what comprises the preparation work and the simulation session, how the two parts are linked and complement each other to support the best learning outcomes. In addition, they should also understand where the simulation session stands within the curriculum. This can be facilitated by fostering collaboration with course directors, key faculty, and other stakeholders. A good understanding of the curriculum and key components of the simulation session helps faculty better envision the flipped simulation, to appreciate the importance of the presimulation activities, and to give clear instructions to students to minimize any confusion. Successful integration of the flipped methodology into simulation and the larger curricula helps faculty and students see the importance and relevance of the simulation.

Professional development is necessary for many to gain the skills needed to successfully develop a flipped simulation session. A key consideration regarding integration into the curriculum is that faculty considers working with experts in education technology; many universities have established centers to help faculty use technology in teaching. Additionally, effective integration requires the faculty and simulation scenario developers to have a good understanding of curriculum development.

\section{Recommendation 5: Applying the Flipped Model to Simulation Requires Buy-in from Faculty, Administration, and Students}

Faculty roles change dramatically in the flipped classroom model. No longer are they the "sage on the stage" but rather they become facilitators of student learning. Different from a typical simulation session, faculty need to take on additional responsibilities when they decide to adopt the flipped classroom approach. In addition to effectively facilitating and debriefing the simulation session, they are responsible for developing the pre-simulation work, holding the students accountable for the work, and making efforts to link preparation work with simulation exercises in debriefing. Research shows that faculty workload with this model increases initially, but there is a significant cost-saving in the long run when faculty repeat the same simulation sessions [30]. Although finding or creating the preparation work can be time consuming, it establishes a transparent knowledge baseline which is a key component to the flipped model. Since several faculty members, oftentimes from different specialties, may be involved in teaching simulation sessions, all faculty involved should complete the same pre-simulation work as the students in order to prepare them for discussing gaps in performance during debriefing. Creating a common understanding of the objectives of the simulation session and the students' knowledge base can promote faculty buy-in and result in less variation between facilitators improving reliability.

Buy-in from the administration is key to the successful integration of the flipped model in simulation. Faculty 
members need the assurance that the administration values integration of the flipped model if they are to commit to the extra time it will take initially. Administrators also have to recognize that the role of the faculty member changes in this model and must acknowledge the faculty effort involved even though no lectures are occurring. Commitment by the administration is needed to assure that there are adequate resources to facilitate faculty adoption of a flipped model for simulation and sustain such efforts.

In addition to a role change for faculty, the student's role in their education changes as well. Successful use of flipped simulation in the curriculum requires students' participation and cooperation $[20,21]$. Students are put in a position in which they are asked to do more self-learning which can be met with some resistance. Although a set of well-developed pre-simulation materials may be available, how students use the materials depends on the faculty members' instructions and students' cooperation to get this accomplished.

The flipped classroom model has been shown repeatedly to enhance student learning as described earlier and we should expect no less when applying this model to simulation. The data is compelling and making it explicit will facilitate buy-in from all the relevant stakeholders.

\section{Conclusion}

In summary, as a primer for simulation educators, we suggest considering 5 recommendations when integrating the flipped classroom model into simulation sessions: (1) The learning objectives should dictate the use of simulation in a flipped model: (2) preparation work should be engaging and optimized by applying techniques emphasized in the flipped classroom approach: (3) students should be held accountable for completion of preparation work: (4) flipped simulation should not stand alone but instead integrate into the larger curricula: and (5) applying the flipped model to simulation requires buyin from faculty, administration, and students.

Many participants in our workshop realized that they had been using the flipped classroom model in simulation but were not aware of the terminology nor ways to strengthen that approach by applying techniques used in the flipped classroom model. We hope that this commentary stimulates discussion among simulation educators to embrace the terms and join the discussion of how flipping the classroom contributes to improved learning outcomes. We anticipate more research will be conducted to study the effectiveness of the flipped simulation model as well as best practices in using the flipped model in healthcare simulation.

Authors' Contributions All authors contributed from the conceptualization of the article, to the writing of the article, and read and approved the final manuscript.

\section{Compliance with Ethical Standards}

Conflict of Interest The authors declare that they have no conflict of interest.

\section{References}

1. 7 things you should know about ... flipped classrooms. EDUCAUSE. 2012.

2. Healthcare Simulation Dictionary. Healthcare simulation dictionary. 2020.

3. Standards Committee INACSL. INACSL standards of best practice: SimulationSM simulation design. Clin Simul Nurs. 2016;12(S):S5-S12.

4. Freeman S, Eddy SL, McDonough M, Smith MK, Okoroafor N, Jordt $\mathrm{H}$, et al. Active learning increases student performance in science, engineering, and mathematics. PNAS USA. 2014;111(23):8410-5

5. Street S, Gilliland K, McNeil C, Royal K. The flipped classroom improved medical student performance and satisfaction in a preclinical physiology course. Medical Science Educator. 2015; 25(1):35-43.

6. Tune JD, Sturek M, Basile D. Flipped classroom model improves graduate student performance in cardiovascular, respiratory, and renal physiology. Advances in Physiology Education. 2013; 37(4):316-320.

7. Wise HH. Student performance and perceptions of a lecture-based course compared with the same course utilizing group discussion. J Phys Ther Educ. 2004;18:75-9.

8. Baker JW. The "classroom flip": using web course management tools to become the guide by the side. In: SELECTED PAPERS FROM THE 11TH INTERNATIONAL CONFERENCE ON COLLEGE TEACHING AND LEARNING; 2000.

9. Stahl G. Theories of cognition in collaborative learning. Int Handb Collab Learn. 2012.

10. Chen F, Lui AM, Martinelli SM. A systematic review of the effectiveness of flipped classrooms in medical education. Med Educ. 2017;51(6):585-97.

11. Johnson GB. Student perceptions of the flipped classroom. Unpublished Master's thesis. 2013.

12. Kachka P. Understanding the flipped classroom: part 2: Faculty Focus, Magna Publications; 2012.

13. Reed T, Pirotte M, McHugh M, Oh L, Lovett S, Hoyt AE, et al. Simulation-based mastery learning improves medical student performance and retention of core clinical skills. Simul Healthc. 2016;11(3):173-80.

14. Reed T, Horsley TL, Muccino K, Quinones D, Siddall VJ, McCarthy J, et al. Simulation using TeamSTEPPS to promote interprofessional education and collaborative practice. Nurse Educ. 2017;42(3):E1-5.

15. Liu KJ, Tkachenko E, Waldman A, Boskovski MT, Hartman RI, Levin AA, et al. A video-based, flipped classroom, simulation curriculum for dermatologic surgery: a prospective, multi-institution study. J Am Acad Dermatol. 2019;81:1271-6.

16. Liebert CA, Lin DT, Mazer LM, Bereknyei S, Lau JN. Effectiveness of the surgery core clerkship flipped classroom: a prospective cohort trial. In: American Journal of Surgery; 2016.

17. Uther P, Van Munster KA, Briggs N, O'Neill S, Kennedy S. Introducing early-phase medical students to clinical paediatrics using simulation and a flipped-classroom. J Paediatr Child Health. 2019;55:1107-12.

18. Chiu HY, Kang YN, Wang WL, Huang HC, Wu CC, Hsu W, et al. The effectiveness of a simulation-based flipped classroom in the 
acquisition of laparoscopic suturing skills in medical students - a pilot study. J Surg Educ. 2018;75:326-32.

19. Riley B. Using the flipped classroom with simulation-based medical education to engage millennial osteopathic medical students. J Am Osteopath Assoc. 2018;118:673-8.

20. Moffett J. Tlve tips for flipping the classroom. Med Teach. 2015;37:331-6.

21. Bilello LA. Turning the tables on tradition: flipped high-Fidelity simulation to potentiate learning. Adv Med Educ Pract. 2019;10: 959-61.

22. Ambrose SA, Bridges MW, DiPietro M, Lovett MC, Norman MK. How learning works: seven research-based principles for smart teaching. how learning works. 2010.

23. Metcalfe J. Is study time allocated selectively to a region of proximal learning? J Exp Psychol Gen. 2002;131(3):349-63.

24. Bloom BS. Taxonomy of educational objectives: the classification of educational goals, book 1: cognitive domain. In: Taxonomy of Educational Objectives; 1956.

25. Cheng A, Eppich W, Grant V, Sherbino J, Zendejas B, Cook DA. Debriefing for technology-enhanced simulation: a systematic review and meta-analysis. Med Educ. 2014;48:657-66.
26. Cook DA. The research we still are not doing: an agenda for the study of computer-based learning. Acad Med. 2005;80(6):541-8.

27. Horsley TL, Reed T, Muccino K. Quinones D. McCarthy J. Developing a foundation for interprofessional education within nursing and medical curricula. Nurse Educ: Siddall VJ; 2016.

28. Van Der Vleuten CPM. The assessment of professional competence: developments, research and practical implications. Adv Health Sci Educ. 1996;1(1):41-67.

29. Szarek JL, Boardman JM, White M, Holt JT. Integrated and flipped: 5 year's experience of integrating active learning in an integrated course. Med Sci Educ. 2016;26:159-67.

30. McLaughlin JE, Roth MT, Glatt DM, Gharkholonarehe N, Davidson CA, Griffin LM, et al. The flipped classroom: a course redesign to foster learning and engagement in a health professions school. Acad Med. 2014;89(2):236-43.

Publisher's Note Springer Nature remains neutral with regard to jurisdictional claims in published maps and institutional affiliations. 